\title{
OPTIMAL ARBITRAGE STRATEGIES ON STOCK INDEX FUTURES UNDER POSITION LIMITS
}

- Min Dai ${ }^{1}$

- Yifei Zhong ${ }^{2}$

- Yue Kuen Kwok ${ }^{3} 4$

Assuming the absence of market frictions, deterministic interest rates, and certainty in dividend payouts from the stocks in the index basket, an arbitrageur can lock in the profit of a positive (negative) arbitrage basis in a stock index futures by adopting a short (long) futures strategy. In addition, the arbitrageur may improve the arbitrage profit by adopting the so-called early unwinding strategy of liquidating the position before maturity, or more aggressively from the long position directly to the short position or vice versa. In this paper, we examine the optimal arbitrage strategies in stock index futures with position limits and transaction costs. In our analysis, the index arbitrage basis is assumed to follow the Brownian Bridge process. The model formulation of the option value functions leads to a coupled system of variational inequalities. We determine the values of the arbitrage opportunities and the optimal threshold values of the arbitrage basis at which the arbitrageur should optimally close an existing position or open a new index arbitrage position. In particular, we examine the impact of transaction costs on the index arbitrage strategies.

The authors would like to thank J.K.W. Fung for his suggestion of the problem and initiation of some of the ideas in the research.

Min Dai would like to acknowledge the support from Singapore MOE ACRF grant (No. R-146-000-096-112) and the National University of Singapore Risk Management Institue grant (No. R-146-000-124-720/646).

Yifei Zhong would like to acknowledge the support from the Oxford-Man Institute of Quantitative Finance grant and NNSF of China (No. 10901060).

\footnotetext{
${ }^{1}$ Min Dai is in the Department of Mathematics at the National University of Singapore, Singapore.

${ }^{2}$ Yifei Zhong is in the Mathematical Institute and Oxford-Man Institute of Quantitative Finance at Oxford University, United Kingdom.

${ }^{3}$ Yue Kuen Kwok is in the Department of Mathematics at the Hong Kong University of Science and Technology, Hong Kong, China.

${ }^{4}$ Correspondence author, Department of Mathematics, Hong Kong University of Science and Technology, Hong Kong, China; email: maykwok@ust.hk.
} 


\section{INTRODUCTION}

It is well known that the replicating portfolio for a forward contract involves a buy-and-hold strategy. Assuming the dividend payouts from the stocks in the portfolio to be deterministic, the forward contract can be fully replicated by the underlying asset. Therefore, the fair value of a forward contract is enforceable by no-arbitrage argument. An exchange-traded futures requires daily settlement through a margin account. Provided that interest rates are non-stochastic (a sufficient condition), the forward price and futures price are equal. Theoretically, the linkage in prices between an index futures and the underlying basket of stocks can be maintained by the actions of the arbitrageurs. In the idealized scenario, these two instruments are perfect substitute of each other. The investors' choice on either one of them will be dictated by transaction costs and convenience. However, various empirical studies reveal that substantial and sustained deviations do exist between the index futures prices and their theoretical values. These differences may arise from order flow imbalance, different estimations of the dividend payouts, transaction costs, and many other factors. The study on the dynamics of stock indexes and the lead-lag relation between the cash market and stock index futures have been well studied in the literature (Stoll and Whaley, 1990; Brennan and Schwartz, 1990; Chan, 1992; Monoyios and Sarno, 2002; Fung and $\mathrm{Yu}, 2007)$.

Index arbitrage can be defined as a strategy designed to profit from temporary discrepancies between the prices of the stocks comprising the index and the futures price on that index. The conventional dynamic hedging strategy of index futures may not be riskfree. For example, there exists risk of uncertainty of dividends or arbitrageurs may be forced out of potentially profitable position due to further widening of the arbitrage basis. Full replication of the basket of stocks may be costly in implementation. The presence of transaction costs may allow the futures prices to fluctuate in a band around the theoretical values. On the other hand, an opposing factor that helps narrowing the band is the arbitrageur's option right to reverse the position prior to expiration.

Let $t$ denote the current time and $T$ be the futures' maturity date. We let $D_{t}$ denote the present value of the dividends paid by the basket of stocks in the index within the life of the futures contract. In our present study, the dividends are assumed to be deterministic. We also assume non-stochastic interest rates so that the index futures and index forward have the same 
price. The time- $t$ value of the $T$-maturity unit par discount bond is denoted by $P_{t}(T)$. We use $S_{t}$ to denote the time- $t$ index value and write $F_{t}(T)$ as the observed time- $t$ price of the $T$-maturity futures. By assuming the absence of market frictions and invoking the standard no-arbitrage argument, the theoretical no-arbitrage futures price would be given by $\left(S_{t}-D_{t}\right) / P_{t}(T)$. As discussed in the above, the observed time- $t$ futures price $F_{t}(T)$ may not agree with this theoretical futures price. We define $G_{t}$ to be

$$
G_{t}=F_{t}(T) P_{t}(T)+D_{t}(T) .
$$

The difference between $G_{t}$ and $S_{t}$ represents the arbitrage profit that can be exploited by arbitrageurs by taking offsetting positions in the index futures and the basket of stocks comprising the index. Accordingly, we define the index arbitrage basis $\epsilon_{t}$ by

$$
\epsilon_{t}=G_{t}-S_{t}
$$

Here, we take a brief review of how to take static arbitrage in an index forward contract if arbitrage basis exists (recall that the arbitrage procedure has to be modified slightly for an index futures due to the dynamic margin requirement). When the basis is positive (negative), the arbitrageur can lock in the profit $\epsilon_{t}\left(-\epsilon_{t}\right)$ by adopting the long (short) arbitrage, which means taking a long (short) position in the underlying basket of stocks and a short (long) position in the futures contract. Specifically, in the long arbitrage strategy, the arbitrageur borrows $G_{t}$ and uses the lesser amount $S_{t}$ to acquire the basket of stocks. The upfront positive cash flow to the arbitrageur is the arbitrage basis $\epsilon_{t}$ (positive value). At the same time, the arbitrageur enters into a short position in futures at no cost. The positions are then being held until the maturity date of the futures and the basket of stocks are delivered to the futures' counterparty at maturity. As a result, the dividends received during the life of the futures and the futures price received at maturity can be used to pay back the loan. Theoretically, index arbitrage opportunities should disappear rapidly once the opportunity can be accomplished without market frictions and many arbitrageurs act on it. However, variability in the index arbitrage basis persists in the financial markets. Noises in trading and market frictions are widely accepted to be the contributing factors for the existence of basis.

The typical market frictions include transaction costs, taxes, and position limits. Usually, there exists a limit on the maximum position that can be taken by an arbitrageur. Since stock index arbitrage involves transaction 
in both the cash stocks and futures markets, finite transaction costs incur due to the presence of commissions and bid-ask spreads in the two markets. Under position limits, the closing of an existing position prior to maturity may provide the possibility of opening of an arbitrage position at a later time on more favorable scenarios. These early unwinding opportunities may help improve the exploitation of arbitrage profit. Our model is an extension of the Brennan-Schwartz model (1990) with the additional flexibility that the arbitrageur is allowed to switch from the long position to the short position directly (or vice versa) without going to the zero position first then to the opposite position at a later time. We observe that the structure of transaction costs plays an important role in determining whether it may be optimal to perform the above direct switching into the opposite position in single transaction. We would like to find the value of the option of closing an existing position or opening a new position when the arbitrageur is subject to position limits and transaction costs.

The paper is organized as follows. In the next section, we present the model setup of the option value of taking an index arbitrage. The option value function of closing or opening position can be formulated as an optimal stopping problem. The underlying force that drives arbitrage is the stochastic dynamics of the arbitrage basis. From empirical observations, arbitrage basis tends to zero as maturity is approached and exhibits the mean reversion property. Following Brennan and Schwartz (1990), the Brownian Bridge process is chosen to model the basis dynamics. The Brownian Bridge process has the desirable property that the arbitrage basis becomes zero at maturity. We derive the coupled system of variational inequalities that govern the option value functions. We then present sample calculations of finding the option values and optimal arbitrage strategies under position limits. We analyze the characteristics of the arbitrage strategies. In the last section, we summarize the results and present conclusive remarks about the index arbitrage strategies on stock index futures under position limits.

\section{MODEL FORMULATION OF OPTION VALUES AS- SOCIATED WITH ARBITRAGE STRATEGIES}

Assuming deterministic dividends, non-stochastic interest rates and the absence of transaction costs, when under the scenario $\epsilon_{t}>0$, an arbitrageur 
can lock in the arbitrage profit $\epsilon_{t}$ by taking a long position in the underlying portfolio, holding it with a short position in the futures contract and holding the position until the maturity date of the futures contract. This is called a simple long arbitrage. It is said to be simple since the position is held until maturity. However, a long or short arbitrage position can be closed out to zero position prior to maturity by taking an offsetting arbitrage position or even converting from the long arbitrage position to the short arbitrage position in a single transaction. Following Brennan and Schwartz (1990), we investigate the value of the option right to close or initiate an arbitrage position under position limits, where an arbitrageur is restricted to single long or short arbitrage position or zero position at any time. With position limit, suppose an arbitrageur is holding the long arbitrage position, closing his current position yields an intermediate arbitrage profit. In addition, he is given the right to initiate a new long or short arbitrage position in the future when the arbitrage basis reaches some sufficiently deep threshold level.

In this section, we construct the model formulation of the option values associated with arbitrage positions under transaction costs and position limits. Let $C_{1}$ and $C_{2}$ denote the associated transaction costs to initiate and close a position (long or short), respectively. Let $C_{3}$ denote the transaction costs to close a long (short) position and initiate the opposite short (long) position in one single transaction. We expect

$$
C_{3}=C_{1}+k C_{2}
$$

where $k \in(0,1]$. When $k=1$, the transaction costs are considered to be proportional. If both fixed and proportional costs are involved in the transactions, then $k<1$.

We use $U(\epsilon, t)$ and $V(\epsilon, t)$ to denote the time- $t$ option value to close a short arbitrage position and long arbitrage position, respectively, and let $W(\epsilon, t)$ denote the time- $t$ value of the option right to initiate an arbitrage position (either long or short position). These options resemble an American option with maturity $T$ coinciding with that of the futures contract. Assuming risk neutrality of the arbitrageur, and supposing the holder is given the exercise right at any time and option's exercise payoff equals $f(\epsilon, t)$, the value of the American style option $P(\epsilon, t)$ is given by

$$
P(\epsilon, t)=\max _{t \leq t^{*} \leq T} E\left[e^{-r\left(t^{*}-t\right)} f\left(\epsilon_{t^{*}}, t^{*}\right) \mid \epsilon_{t}=\epsilon\right],
$$

where $t^{*} \in[t, T]$ is the optimal stopping time, $r$ is the riskless interest rate, and $E$ is the expectation under the physical measure. 
To close a short arbitrage position, we can either take one offsetting long arbitrage position or two long arbitrage positions, the immediate realized arbitrage profit is $\epsilon$ or $2 \epsilon$, while the transaction costs are $C_{2}$ or $C_{3}$, respectively. The latter case corresponds to the direct conversion from the short position to the long position in one single transaction. Also, it opens the right to initiate a new arbitrage position or close a long arbitrage position in the future. Accordingly, the corresponding exercise payoff associated with the option value $U(\epsilon, t)$ is given by taking the maximum value among the above two payoffs

$$
\max \left\{\left(W(\epsilon, t)+\epsilon-C_{2}\right)^{+},\left(V(\epsilon, t)+2 \epsilon-C_{3}\right)^{+}\right\},
$$

where the notation $x^{+}$denotes $\max (x, 0)$. In a similar manner, the exercise payoff associated with $V(\epsilon, t)$ is

$$
\max \left\{\left(W(\epsilon, t)-\epsilon-C_{2}\right)^{+},\left(U(\epsilon, t)-2 \epsilon-C_{3}\right)^{+}\right\} .
$$

With no position at the current time, the arbitrageur can initiate a long or short arbitrage position with transaction cost $C_{1}$. The respective realized arbitrage profit is $\epsilon$ or $-\epsilon$ together with the option right to close the position in the future. The exercise payoff associated with $W(\epsilon, t)$ is seen to be

$$
\max \left\{\left(V(\epsilon, t)+\epsilon-C_{1}\right)^{+},\left(U(\epsilon, t)-\epsilon-C_{1}\right)^{+}\right\} .
$$

\section{Dynamics of the arbitrage basis}

To evaluate the option value functions $U, V$ and $W$, it is necessary to prescribe the stochastic dynamics of the arbitrage basis $\epsilon_{t}$. Following Brennan and Schwartz (1990), the evolution of $\epsilon_{t}$ under the physical measure is governed by the Brownian Bridge process as follows:

$$
d \epsilon_{t}=-\frac{\mu \epsilon_{t}}{T-t} d t+\gamma d W_{t}
$$

where $\mu$ is the speed of mean reversion, $\gamma$ is the instantaneous standard deviation of the process, and $W_{t}$ is the standard Brownian process. Here, we take $\mu$ and $\gamma$ to be positive constants. The assumption of constancy of the mean reversion speed parameter $\mu$ is not without criticism. For example, one may expect $\mu$ to be dependent on the order imbalance between buying and selling orders in the cash stock market, which is commonly considered 
as the proxy of the market consensus of the future directional move of the stock index (Fung and Yu, 2008).

The Brownian Bridge process has the desirable property that it is tied down to zero at maturity almost surely. As $t \rightarrow T^{-}$, the speed of mean reversion to zero value is very large, so $\epsilon_{t}$ goes quickly towards its mean level (which is zero).

\section{Variational inequalities formulation}

Since index arbitrage is considered in our model, the commonly adopted no-arbitrage argument cannot be applied to derive the pricing formulation of these embedded options. We follow the same pricing approach adopted in the Brennan-Schwartz model (1990), where the values of the options are determined by discounting their expected payoffs at the riskless interest rate $r$. It can be argued that this assumption is consistent with risk neutrality of the arbitrageur. An alternative justification to the pricing approach is that we may assume a representative individual model in which innovations in arbitrage basis $\epsilon_{t}$ are uncorrelated with those in aggregate consumption in the financial market. Given the dynamics of $\epsilon_{t}$, both $U, V$ and $W$ satisfy the same governing differential equation in their respective continuation regions and their values equal to the corresponding exercise payoff in their respective exercise regions. Similar to an American option model, the option value function is governed by a set of variational inequalities (Kwok, 2008). Since the exercise payoff of $U$ involves $V$ and $W$, and similar dependence on other value functions is exhibited in $V$ and $W$ also, the corresponding variational inequalities formulation forms a coupled system between these three option value functions.

Let $\tau=T-t$ denote the time to maturity. The evaluation of the option value functions $U, V$ and $W$ requires the solution of the following coupled system of variational inequalities:

$$
\begin{aligned}
\min \left\{\frac{\partial U}{\partial \tau}-\frac{\gamma^{2}}{2} \frac{\partial^{2} U}{\partial \epsilon^{2}}+\frac{\mu \epsilon}{\tau} \frac{\partial U}{\partial \epsilon}+r U,\right. \\
\left.U-\max \left[\left(W(\epsilon, t)+\epsilon-C_{2}\right)^{+},\left(V(\epsilon, t)+2 \epsilon-C_{3}\right)^{+}\right]\right\}=0 \\
\min \left\{\frac{\partial V}{\partial \tau}-\frac{\gamma^{2}}{2} \frac{\partial^{2} V}{\partial \epsilon^{2}}+\frac{\mu \epsilon}{\tau} \frac{\partial V}{\partial \epsilon}+r V,\right. \\
\left.V-\max \left[\left(W(\epsilon, t)-\epsilon-C_{2}\right)^{+},\left(U(\epsilon, t)-2 \epsilon-C_{3}\right)^{+}\right]\right\}=0
\end{aligned}
$$




$$
\begin{aligned}
\min \{ & \frac{\partial W}{\partial \tau}-\frac{\gamma^{2}}{2} \frac{\partial^{2} W}{\partial \epsilon^{2}}+\frac{\mu \epsilon}{\tau} \frac{\partial W}{\partial \epsilon}+r W \\
& \left.W-\max \left[\left(V(\epsilon, t)+\epsilon-C_{1}\right)^{+},\left(U(\epsilon, t)-\epsilon-C_{1}\right)^{+}\right]\right\}=0
\end{aligned}
$$

It is relatively straightforward to check that

$$
U(\epsilon, t)=V(-\epsilon, t) \quad \text { and } \quad W(\epsilon, t)=W(-\epsilon, t) .
$$

To avoid the singularity at $\tau \rightarrow 0^{+}$in the first order derivative term in the governing differential equation, we adopt the following transformation of independent variables:

$$
x=\tau^{-\mu} \epsilon \quad \text { and } \quad s=\frac{1}{1-2 \mu} \tau^{1-2 \mu},
$$

assuming $\mu<\frac{1}{2}$. The governing differential equation can be simplified to become

$$
\frac{\partial U}{\partial s}-\frac{\gamma^{2}}{2} \frac{\partial^{2} U}{\partial x^{2}}+r[(1-2 \mu) s]^{\frac{2 \mu}{1-2 \mu}} U=0
$$

Remark

In the Brennan-Schwartz model (1990), the arbitrageur is not allowed to switch from the long position directly into the short position or vice versa in a single transaction. Under their framework, the above variational inequalities formulation reduces to

$$
\begin{gathered}
\min \left\{\frac{\partial U}{\partial \tau}-\frac{\gamma^{2}}{2} \frac{\partial^{2} U}{\partial \epsilon^{2}}+\frac{\mu \epsilon}{\tau} \frac{\partial U}{\partial \epsilon}+r U, U-\left(W(\epsilon, t)+\epsilon-C_{2}\right)^{+}\right\}=0 \\
\min \left\{\frac{\partial V}{\partial \tau}-\frac{\gamma^{2}}{2} \frac{\partial^{2} V}{\partial \epsilon^{2}}+\frac{\mu \epsilon}{\tau} \frac{\partial V}{\partial \epsilon}+r V, V-\left(W(\epsilon, t)-\epsilon-C_{2}\right)^{+}\right\}=0 \\
\min \left\{\frac{\partial W}{\partial \tau}-\frac{\gamma^{2}}{2} \frac{\partial^{2} W}{\partial \epsilon^{2}}+\frac{\mu \epsilon}{\tau} \frac{\partial W}{\partial \epsilon}+r W\right. \\
\left.W-\max \left[\left(V(\epsilon, t)+\epsilon-C_{1}\right)^{+},\left(U(\epsilon, t)-\epsilon-C_{1}\right)^{+}\right]\right\}=0
\end{gathered}
$$

The numerical solution of the coupled system of variational inequalities (5) can be effectively obtained using finite difference calculations combined with the use of the Projected-Successive-Over-Relaxation (PSOR) method 
(Kwok, 2008). We adopt an implicit discretization of the coupled differential equations in the finite difference scheme. The solution of the resulting algebraic system of equations for the option values is then solved by the GaussSeidel iterative scheme. In each of the Gauss-Seidel iterative step in finding the numerical approximation of the option values in the next iteration, we apply the dynamic programming procedure of taking the maximum value among the exercise payoff and the continuation value. The exercise payoff values can be conveniently computed based on the option values obtained in the previous iteration.

\section{SAMPLE CALCULATIONS ON OPTIMAL ARBITRAGE STRATEGIES}

We performed the sample calculations to find the option value functions $U, V$ and $W$. The optimal threshold values for the arbitrage basis at which the arbitrageur should optimally choose to change from one state of position to another state are also obtained as part of the solution of the optimal stopping model. We let $\epsilon_{W}^{*}(t)$ and $\epsilon_{V}^{*}(t)$ denote the exercise boundaries for the option price functions $W$ and $V$, respectively. In our numerical calculations, we choose the parameter values: $\gamma=0.6, \mu=0.3, r=0.07, T=1, C_{1}=$ $1.2, C_{2}=0.5$ and $C_{3}=1.5$. In Figure 1 , we show the plot of the value functions $U(\epsilon, t), V(\epsilon, t)$ and $W(\epsilon, t)$ at $t=0$ as a function of $\epsilon$. The plots verify the symmetric properties of the value functions as stated in eq. (6). Obviously, $U(\epsilon, t)$ assumes a small value where $\epsilon$ is negative since it is much unlikely to close the short arbitrage position by taking the long arbitrage position when the arbitrage basis is negative. Also, $W$ should have a very small value when $|\epsilon|$ is less than the transaction cost to initiate an arbitrage position.

The optimal arbitrage strategies can be revealed by finding the critical threshold values $\epsilon_{W}^{*}(t)$ and $\epsilon_{V}^{*}(t)$ for the arbitrage basis so that one can identify the continuation region and stopping region of $W$ and $V$. In Figure 2, we show the plot of $\epsilon_{W}^{*}(t)$ and $\epsilon_{V}^{*}(t)$ against $t$ [by symmetry, $\epsilon_{U}^{*}(t)=-\epsilon_{V}^{*}(t)$ ]. The upper and lower solid curves show the two branches of $\epsilon_{W}^{*}(t)$. When $\epsilon_{t}$ lies above the upper branch of $\epsilon_{W}^{*}(t)$, the arbitrageur who is holding the zero position should initiate a long arbitrage position (as signified by $W \rightarrow V$ in Figure 2). By symmetry, when $\epsilon_{t}$ lies below the lower branch of $\epsilon_{W}^{*}(t)$, 
the arbitrageur should optimally swap from the zero position to the short arbitrage position $(W \rightarrow U)$. As $t \rightarrow T^{-},\left|\epsilon_{W}^{*}\left(T^{-}\right)\right|$tends to the value that equals the transaction cost $C_{1}$ required for initiating an arbitrage position. The upper (lower) branch of $\epsilon_{W}^{*}(t)$ decreases (increases) with an increasing value of $t$ since a higher absolute value of the arbitrage basis is required to initiate an arbitrage position with a higher value of time to maturity $\tau$. As $\epsilon_{t}$ is tied down to the zero value as $t \rightarrow T^{-}$, it is likely that there will be no change in the position as time is approaching maturity.

When the arbitrageur is currently holding a long arbitrage position, he can either close the long arbitrage position to the zero position $(V \rightarrow W)$ or convert directly to the short arbitrage position $(V \rightarrow U)$. The latter case occurs when the arbitrage basis is sufficiently deep in negative value [see the lower dotted curve $\epsilon_{V}^{*}(t)$ in Figure 2]. In our sample calculations, we have chosen $C_{3}<C_{1}+C_{2}$. With a lower value of single trip transaction cost $C_{3}$ as compared to the sum of two transaction costs $C_{1}+C_{2}$, the arbitrageur has a higher incentive to choose the direct conversion to the opposite arbitrage position $(V \rightarrow U)$ instead of taking $V \rightarrow W$ first and $W \rightarrow U$ subsequently. When the time to maturity becomes shorter, it is now optimal for the arbitrageur to close the long position to the zero position (that is, $V \rightarrow W$ ) at some threshold value of the arbitrage basis which is less deep (in negative value) when compared with that required for the direct conversion $V \rightarrow U$. The stopping region of $V$ within which it is optimal to take the action $V \rightarrow W$ is the region bounded by the two dotted curves shown in Figure 2.

What would happen when $C_{3}=C_{1}+C_{2}$, that is, there is no savings on the transaction costs in taking a single transaction into the opposite position directly instead of taking the sequential two-step transactions. We performed similar calculations with the same set of parameter values as those used for plotting Figure 2, except that $C_{3}=C_{1}+C_{2}=1.7$. The new set of optimal exercise boundaries $\epsilon_{V}^{*}(t)$ and $\epsilon_{W}^{*}(t)$ are plotted against time $t$ in Figure 3. We observe that the exercise region corresponding to $V \rightarrow U$ does not appear and the exercise boundary for $V \rightarrow W$ becomes one single curve (shown as the dotted curve in Figure 3). Suppose $C_{3}=C_{1}+C_{2}$, when the arbitrageur is currently in the long position, he chooses to convert optimally from the long position into the zero position when $\epsilon$ falls in negative value to the exercise boundary $\epsilon_{V}^{*}(t)$ (dotted curve) and from the zero position into the short position at a later time instant when $\epsilon$ falls further in negative value to the exercise boundary $\epsilon_{W}^{*}(t)$ (lower solid curve). These new results under 
$C_{3}=C_{1}+C_{2}$ reveal that our optimal stopping model as governed by the set of variational inequalities in (5) reduces to the Brennan-Schwartz model shown in (7). To establish the above statement mathematically, let $U, V$ and $W$ be the solution to the system in (7). It suffices to show that

$$
U(\epsilon, t) \geq\left(V(\epsilon, t)+2 \epsilon-C_{3}\right)^{+}
$$

and

$$
V(\epsilon, t) \geq\left(U(\epsilon, t)-2 \epsilon-C_{3}\right)^{+} .
$$

To show inequality (8a), we consider

$$
\begin{aligned}
U(\epsilon, t) & \geq W(\epsilon, t)+\epsilon-C_{2} \\
& \geq V(\epsilon, t)+\epsilon-C_{1}+\epsilon-C_{2} \\
& =V(\epsilon, t)+2 \epsilon-\left(C_{1}+C_{2}\right) \\
& =V(\epsilon, t)+2 \epsilon-C_{3} .
\end{aligned}
$$

Inequality $(8 \mathrm{~b})$ can be established in a similar manner. Therefore, the option value functions $U, V$ and $W$ also satisfy the system in (5). By virtue of uniqueness of solution, the two systems of variational inequalities shown in (5) and (7) are equivalent.

Our model formulation can be extended from one position limit to multiple position limits, say, the number of positions $i$ can be $n, n-1, \cdots, 1,0,-1$, $\cdots,-n$, where $n>1$. The general model formulation should allow the arbitrageur to change his position from $i$ to $j$, where $i$ and $j$ can be any integer between $-n$ and $n$. The amount of computational effort only grows linearly with the number of positions. In summary, the Brennan-Schwartz formulation allows the following transactions in the position limit: $-1 \rightarrow 0,0 \rightarrow$ $1,1 \rightarrow 0,0 \rightarrow-1$ while our model in (5) allows the additional flexibility of taking $-1 \rightarrow 1$ and $1 \rightarrow-1$, provided that there exist savings in the transaction costs in taking direct conversion from the long to short or the short to long.

\section{CONCLUSION}

We construct an optimal stopping model that analyzes the optimal index arbitrage strategies in stock index futures with position limits and transaction 
costs. Instead of adopting the simple arbitrage strategy where the arbitrage position is held until the maturity date of the futures contract, we examine the option value associated with the long or short arbitrage position which can be closed out or converted into the opposite position prior to maturity. By solving a coupled system of variational inequalities that govern these option value functions, we obtain the optimal arbitrage basis threshold level at which it is optimal for the arbitrageur to change from one arbitrage position to another position. Depending on the arbitrage basis level, time to maturity and transaction costs structure, the arbitrageur may choose to close the long position to the zero position or convert from the long into short position in a single transaction. This index arbitrage model provides an interesting example of analyzing the associated optimal stopping policies that allow an interchange between the multiple states in the system.

\section{BIBLIOGRAPHY}

Brennan, M., Schwartz, E., (1990). Arbitrage in stock index futures. Journal of Business 63(1), S7-S31.

Chan, K., (1992). A further analysis of the lead lag relation between the cash market and stock index futures market. Review of Financial Studies, 5, 123-152.

Fung, J.K.W., Yu, P.L.H., (2008). Order imbalance and the dynamics of index and futures prices. Journal of Futures Markets, 27(12), 1129-1157.

Kwok, Y.K., (2008). Mathematical Models of Financial Derivatives, second edition, Springer.

Monoyios, M., Sarno, L., (2002). Mean reversion in stock index futures markets: a nonlinear analysis. Journal of Futures Markets, 22(4), 285-314.

Stoll, H.R., Whaley, R.E., (1990). The dynamics of stock index and stock index futures return. Journal of Financial and Quantitative Analysis, 25, 441-468. 


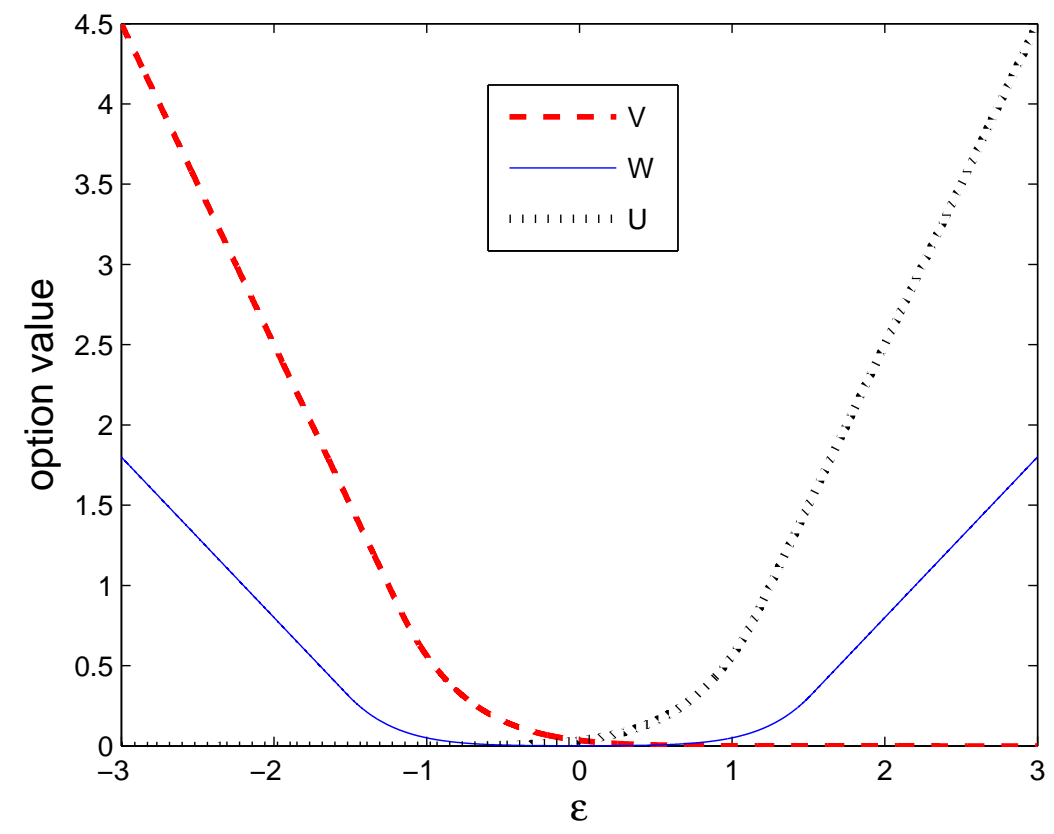

Figure 1 Option value functions $U, V$ and $W$ are plotted against arbitrage basis $\epsilon$ at $t=0$. We observe that $W$ is an even function in $\epsilon$, and the pair of the value functions $U$ and $V$ are symmetric about the vertical line $\epsilon=0$. When the absolute value of arbitrage basis is smaller than the transaction cost of opening a new position, the option value $W$ has an insignificantly small value. 


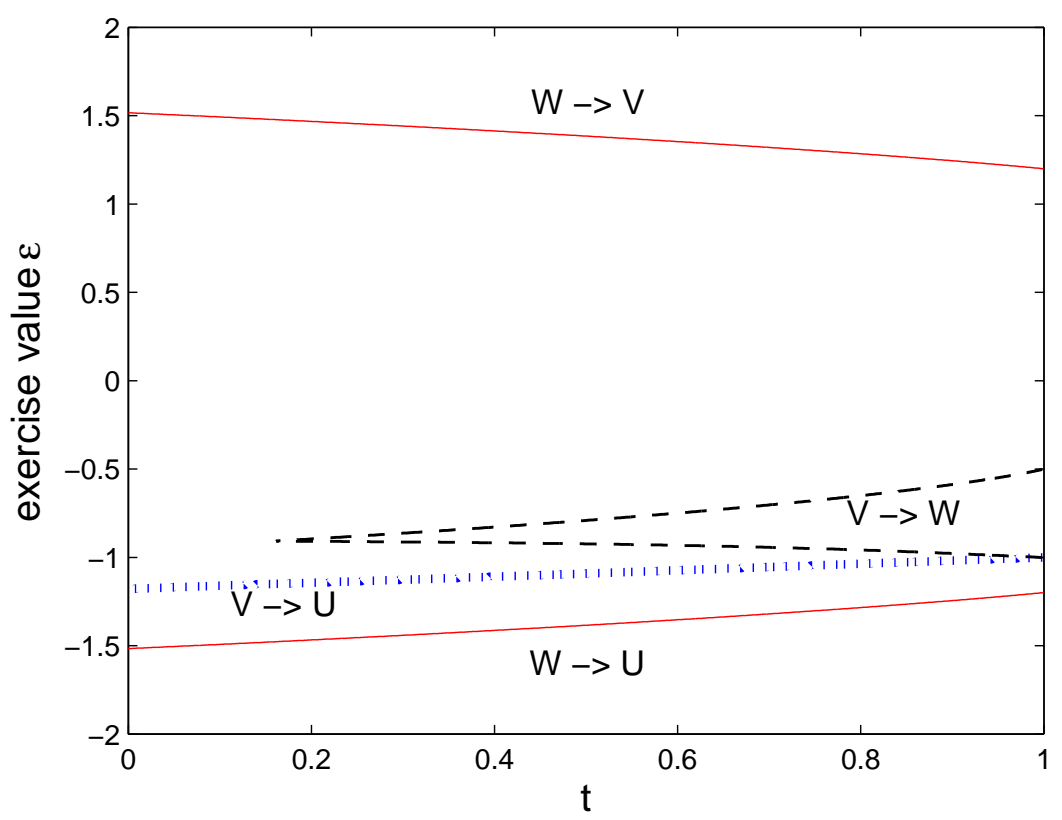

Figure 2 The optimal exercise boundaries $\epsilon_{V}^{*}(t)$ and $\epsilon_{W}^{*}(t)$ of $V$ and $W$ are plotted against time $t$, assuming $C_{3}<C_{1}+C_{2}$. There are two branches of $\epsilon_{W}^{*}(t)$ (shown by the two solid curves) which end at $\epsilon_{W}^{*}(1)= \pm 1.2$. When the arbitrage basis $\epsilon$ stays above (below) the upper (lower) branch, it is optimal to open the long (short) arbitrage position corresponding to $W \rightarrow V(W \rightarrow U)$. When the arbitrageur is currently in the long position, it may be optimal to close the long position $(V \rightarrow W)$ when $\epsilon$ falls within the region bounded by the two dashed curves or convert from the long to short position $(V \rightarrow U)$ when $\epsilon$ falls below the dotted curve. 


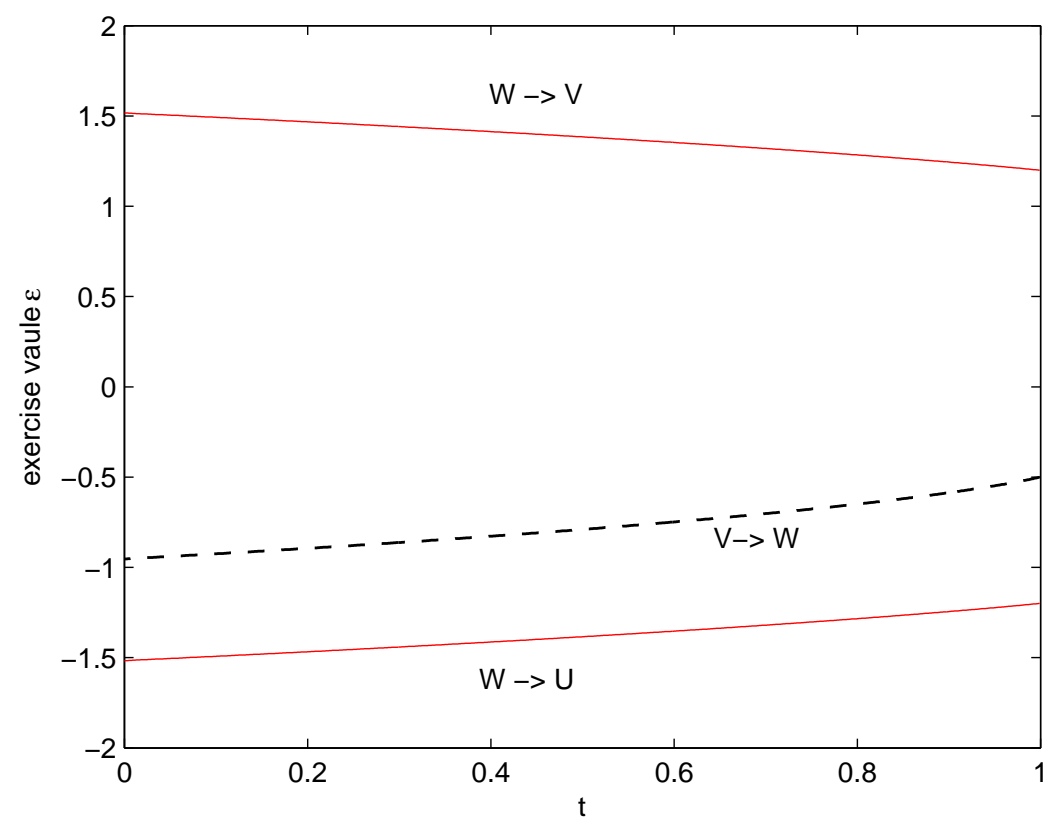

Figure 3 The optimal exercise boundaries $\epsilon_{V}^{*}(t)$ (shown as the dotted curve) and $\epsilon_{W}^{*}(t)$ (shown as the solid curves) of $V$ and $W$ are plotted against $t$, assuming $C_{3}=C_{1}+C_{3}$. The exercise region corresponding to $V \rightarrow U$ does not appear since there are no savings in transaction costs associated with the direct conversion from the long position into the short position in a single transaction. The conversion from $V \rightarrow U$ has to be accomplished in two sequential steps: $V \rightarrow W$ then $W \rightarrow U$ in response to falling value in the arbitrage basis $\epsilon$. 